\title{
A Hierarchical Multi-hop Multimedia Routing Protocol for Wireless Multimedia Sensor Networks
}

\author{
Denis Rosário ${ }^{1}$, Rodrigo Costa ${ }^{2}$, Helder Paraense ${ }^{3}$, Kássio Machado ${ }^{4}$, \\ Eduardo Cerqueira $^{5}$, Torsten Braun ${ }^{6}$, Zhongliang Zhao ${ }^{7}$ \\ 1, 2, 3, 5 Federal University of Pará \\ Rua Augusto Corrêa 01, Belém (Brazil) \\ ${ }^{4}$ Federal University of Minas Gerais \\ Av. Antônio Carlos, 6627, Belo Horizonte (Brazil) \\ ${ }^{1,6,7}$ Institute of Computer Science and Applied Mathematics, University of Bern \\ Neubrückstrasse 10, Bern (Switzerland) \\ E-mail: ${ }^{1}$ denis@ufpa.br, ${ }^{2}$ rodrigomc@ufpa.br, ${ }^{3}$ helderp@ufpa.br, ${ }^{4}$ kassiolsm@ufmg.br, \\ ${ }^{5}$ cerqueira@ufpa.br, ${ }^{6}$ braun@iam.unibe.ch, ${ }^{7}$ zhao@iam.unibe.ch
}

Received: July 31, 2012 Accepted: November 11, 2012 Published: December 16, 2012 DOI: $10.5296 / n p a . v 4 i 4.2121$

URL: http://dx.doi.org/10.5296/npa.v4i4.2121

\begin{abstract}
Wireless Multimedia Sensor Networks (WMSNs) play an important role in pervasive and ubiquitous systems. WMSNs promise a wide scope of potential applications in both civilian and military areas, which require visual and audio information such as environmental monitoring, smart parking, traffic control, and other applications for smart cities. The multimedia content in such applications has the potential to enhance the level of collected information, show the real impact of the event and help to detect objects or intruders. However, WMSN applications must assure reliability, scalability, energy-efficiency and quality level (also from the user's point-of-view) to support the transmission of multimedia content. With this goal in mind, this article outlines a smart Multi-hop hierarchical routing protocol for Efficient VIdeo communication over WMSN (MEVI). MEVI combines a cluster formation scheme with a minimal signaling overhead, a cross-layer solution to select routes based on network conditions and energy issues, and a smart scheme to trigger multimedia transmission according to sensed data. The cluster approach aims to minimize the energy consumption and is suitable for the distribution of multimedia content in WMSNs.
\end{abstract}


Simulation experiments were conducted to show the benefits of MEVI in disseminating video content compared with Low-Energy Adaptive Clustering Hierarchy (LEACH) and Power Efficient Multimedia Routing (PEMuR) in terms of network lifetime and video quality level.

Keywords: Hierarchical architecture, Multimedia content, Routing protocol, Scalability, Video quality, Wireless multimedia sensor networks.

\section{Introduction}

The proliferation of multimedia applications and the demand for new audio/video services in Wireless Sensor Networks (WSNs) [1] are creating a new multimedia sensorbased era, and have fostered the development of Wireless Multimedia Sensor Networks (WMSNs) [2]. For environmental monitoring, video surveillance, traffic control and smart cities applications, multimedia content provides more precise information than simple scalar data, enabling the end user (or system) to visually verify the real impact of the event, take consciousness of what is happening in the environment, plan actions according to these visual information and help to detect objects or intruders or/and analyzing scenes.

The multimedia data collected by the sensor nodes needs to be sent to the Base Station (BS) with a low delay and loss rate, to enable end-users (or systems) to take efficient actions. However, the transmission of multimedia content (video streaming, images or audio) over WMSNs requires real-time delivery and high bandwidth. Hence, it adds more constraints on the design of routing protocols for WMSNs meet energy-efficiency, scalability, reliability, and at the same time, provides Quality of Service (QoS) and Quality of Experience (QoE) assurance for multimedia data [3].

Routing protocols for WMSNs can be classified into flat, location-based and hierarchical ones [4]. In the meantime, for WMSNs a hierarchical architecture has proven to be more beneficial than flat architectures in terms of less energy consumption, higher functionality, better scalability and reliability [2], [4]. The advantages of using a hierarchical architecture are as follows: i) the nodes have different roles or functionalities to reduce energy consumption; ii) the Cluster-Head $(\mathrm{CH})$ performs data aggregation, which avoids unnecessary data transmission; and iii) the non-CH can turn off the radio after transmitting its packets, reducing energy consumption and avoiding communication conflicts.

Another key issue for routing protocols in WMSNs is the multiple hop capability to increase the scalability of the system. However, route selection should select reliable routes based on cross-layer information, i.e. parameters from other protocol layers are taken into account to improve network performance [4]. This requires that nodes must be able to perceive network conditions, have knowledge of the remaining energy of neighbor nodes, and the number of hops to reach the destination node for each possible path. Then, the nodes should dynamically plan, adapt and take appropriate actions for routing decision. Thus, it is possible to select reliable routes based on cross-layer information, which can increase reliability and assure video distribution with quality level support. 
Additionally, energy constraints in WMSNs are even stricter than in WSNs, due to the fact that multimedia content creates a huge amount of data that has to be processed, transmitted and forwarded. The nodes should also be able to sense environmental conditions, take consciousness of what is happening in the environment, and thus take appropriate decisions for multimedia retrieval.

The main requirement for an energy-efficient hierarchical routing protocol in WMSNs is to minimize the signaling overhead for cluster formation and trigger multimedia retrieval and transmission only in case of special events. At the same time, by using multiple hops and a cross-layer approach (e.g., network conditions, energy and hop count), the routing protocol is able to select QoS/QoE-aware routes to transmit multimedia and scalar data, and thus provide scalability and reliability. However, current hierarchical routing protocols for WMSNs do not take into account such key characteristics [2], [4].

To address these questions of higher signaling overhead for cluster formation, reliable cross-layer schemes for routing selection and energy-efficiency, this paper presents an extended version of a smart Multi-hop hierarchical routing protocol for Efficient VIdeo communication over WMSNs (MEVI), which was originally proposed in [5]. MEVI aims to overcome the drawbacks that have been discussed before and allow the transmission of multimedia content with QoS/QoE support by introducing a hierarchical routing protocol with a cluster formation with low signaling overhead, a cross-layer scheme for route selection, and operational modes to trigger multimedia transmission.

This paper extends our previous work by considering the sensing range of camera nodes defined by a Field-of-View (FoV) and not by a disk model. As soon as an event has been detected, the camera node must change its FoV to the area of the event with the aim to retrieve multimedia information from the exact area of the event. Moreover, with the help of a FoV, it is possible to define a subset of camera nodes that can cover a given area. Additionally, this paper considers the weighted arithmetic average to compute the link quality for cluster formation, which gives more importance to recent link quality values. New experiments are also presented in the paper, to show the impacts and benefits of MEVI proposed in this paper for dissemination of video content. MEVI was compared with PEMuR, two versions of LEACH, and two versions of MEVI in terms of network lifetime and video quality level.

The main advantages of MEVI are the following, MEVI (i) implements a mechanism for cluster formation with low overhead, which involves only sending beacon messages; (ii) provides a multi-hop communication between $\mathrm{CHs}$ and BS to assure scalability; (iii) uses a cross-layer scheme to perceive the network conditions and select routes based on link quality and energy parameters; (iv) makes use of the sensed physical environmental conditions from scalar sensor nodes to help the camera nodes to take appropriate decisions with regard to video retrieval and transmission; and (v) enables the camera node to change the FoV to retrieve multimedia content from the event area.

Simulations were carried out to show the impact and benefits of MEVI for dissemination of video content in WMSNs, and make a comparison with LEACH and PEMuR protocols in 
large and small scale scenarios. This paper includes an analysis of energy-efficiency, overhead and video quality. The last of these was analyzed by means of well-known QoE objective metrics, which are Structural Similarity (SSIM) and Video Quality Metric (VQM) $[6]$.

The remainder of this article is structured as follows. Section 2 outlines the related hierarchical routing protocols and their drawbacks. Section 3 describes the proposed smart multi-hop hierarchical routing protocol for efficient video communication over WMSN. The simulations and results are shown in Section 4. Section 5 summarizes the main contributions and results of this paper.

\section{Related Work}

Several efforts have been made to achieve promising results in hierarchical routing protocols for WMSNs, where their main objectives are to minimize energy consumption but achieving a certain video quality level from the user perspective. To achieve these goals, the proposed hierarchical routing protocols make use of multi-hop communication, ant-colonies, social networks, power allocation or video packet scheduling as it will be described in this section.

Low-Energy Adaptive Clustering Hierarchy (LEACH) [7] is the main precursor of a hierarchical routing protocol for WSNs. It achieves low energy dissipation and latency without sacrificing application-specific quality for WSNs. Periodically, nodes perform cluster formation, $\mathrm{CH}$ election and data collection. LEACH forms clusters by using a distributed scheme, which includes beacon, join and schedule messages. The main drawback of LEACH is that it depends on a single-hop communication between $\mathrm{CHs}$ and $\mathrm{BS}$, which is not suitable for large-scale WMSNs as expected for future networks. Periodic data transmissions of multimedia data are unnecessary, since they cause an ineffective expenditure of energy. Moreover, LEACH generates a high signaling overhead for cluster formation, which decreases the network lifetime and consumes scarce sensor node resources.

Ant-colony routing protocols for WMSNs were proposed in [8], [9]. An Ant-based Service-Aware Routing algorithm (ASAR) [8] aims to maximize the use of network resources and to improve network performance. ASAR takes into account three different types of services (event-driven, data and stream query), and four QoS requirements (latency, packet loss, energy consumption and bandwidth). Moreover, AntSensNet [9] has been proposed to achieve QoS for multimedia applications. This protocol builds a hierarchical network architecture and chooses a path that meets QoS requirements for maximizing network utilization. Furthermore, it uses efficient multi-path video packet scheduling to achieve minimum video distortion transmission. However, these ant-colony protocols do not evaluate the quality level of the transmitted multimedia content from the user's perspective. They also exchange a large number of control messages for route discovery, which increases the energy consumption and reduces network lifetime. Finally, the solutions of ant-colonies have not been explored in real applications, because they require a long time to react to topology changes and need a large number of messages for route discovery. 
Adaptive Reliable routing based on Cluster Hierarchy for wireless multimedia sensor network (ARCH) [10] aims to balance energy consumption and obtain the required system reliability. ARCH proposed a power allocation mechanism to adjust the transmission power of nodes, and an energy prediction mechanism. Energy Efficiency QoS Assurance Routing in Wireless Multimedia Sensor Networks (EEQAR) [11] introduces a social network analysis to optimize network performance. EEQAR focuses on how to build energy-efficient QoS assurance routing for WMSNs. However, ARCH and EEQAR do not use a link quality estimator to select reliable routes, which is important for enabling nodes to estimate a value for the communication quality level between the neighbors and thus increase reliability, as shown in [3]. In addition, they include an extra overhead in the route discovery process for intra-cluster communications. Finally, they do not evaluate the video quality level from the user perspective as expected for many WMSN applications.

The Power Efficient Multimedia Routing (PEMuR) protocol [12] is designed to provide an efficient video communication based on a combination of a hierarchical routing protocol and a video packet scheduling model. It creates clusters in a centralized way by using beacon, schedule, advertisement, identifier and join messages. The main drawback of PEMuR is that it only uses the remaining energy to find routes (not link quality), which makes the protocol unreliable for multi-hop transmission and thus does not assure the transmission of videos with QoS/QoE support. PEMuR relies on a centralized way to create clusters, which assumes that the BS is able to communicate with all the nodes by using single-hop communication, which is not realistic for large-scale sensor networks. Additionally, PEMuR includes high signaling overhead, which decreases network lifetime.

Table 1 - Comparison between routing protocols for WMSNs

\begin{tabular}{|c|c|c|c|}
\hline Parameters & Multimedia transmission & Overhead & Route selection \\
\hline LEACH & Periodically & High overhead for cluster formation & Single-hop \\
\hline ASAR & Event or query based & $\begin{array}{c}\text { Ant-base protocol require high number } \\
\text { of messages for route discovery }\end{array}$ & QoS Metrics \\
\hline AntSensNet & Periodically or Event based & $\begin{array}{c}\text { Ant-base protocol require high number } \\
\text { of messages for route discovery }\end{array}$ & QoS Metrics \\
\hline ARCH & Periodically & $\begin{array}{c}\text { Overhead for route discovery process for } \\
\text { intra-cluster communications }\end{array}$ & QoS Metrics \\
\hline EEQAR & Periodically & $\begin{array}{c}\text { Overhead for route discovery process for } \\
\text { intra-cluster communications }\end{array}$ & QoS Metrics \\
\hline PEMuR & Periodically & High overhead for cluster formation & Residual energy \\
\hline
\end{tabular}

It is evident from analyzing the related work that multi-hop communication with a crosslayer mechanism to select routes is required to enhance the video quality level without increasing the network overhead, and thus to reduce the network resource usage. Additionally, the hierarchical routing protocol should create low overhead for cluster formation. Finally, the multimedia transmissions should be triggered only in case of events. 


\section{Macrothink Institute}

However, the current hierarchical routing protocols do not take into account all of these important characteristics into a single proposal that can support a QoE-aware multimedia transmission and energy-efficiency. Table 1 summarizes the main problems for each protocol.

\section{A Multi-hop hierarchical routing protocol for Efficient VIdeo communication over WMSNs (MEVI)}

This section outlines a smart Multi-hop hierarchical routing protocol for Efficient VIdeo communication over WMSNs (MEVI). MEVI proposes: (i) a cluster formation with low overhead; (ii) multi-hop communication with a cross-layer mechanism to select routes based on perceived network conditions; and (iii) operational modes to trigger multimedia transmission according to sensed physical environmental information.

\subsection{Protocol Model Description}

MEVI relies on a hierarchical network architecture with heterogeneous nodes, expected for typical WMSN scenarios and recommended by Almalkawi et al. [2], to reduce the overall communication overhead, maximize network lifetime, and improve scalability and reliability of the system. The nodes have heterogeneous capabilities and are divided into the following classes: (i) scalar sensor nodes, restricted in terms of energy supply, processing and memory; and (ii) camera nodes, equipped with a richer energy source, video camera as well as larger memory and processing capabilities. The network architecture is depicted in Figure 1.
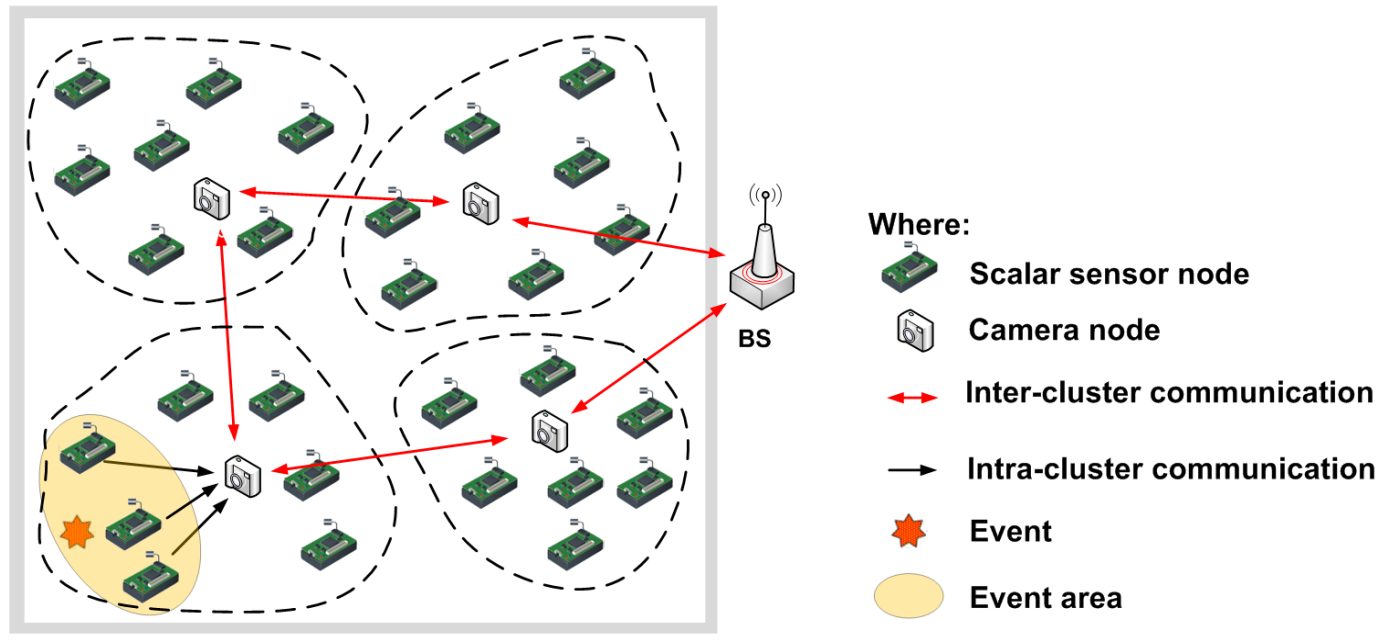

Figure 1 - Network architecture

As mentioned before, the energy constraints in WMSNs are even stricter than in WSNs, because multimedia content creates a huge amount of data that has to be processed, transmitted and forwarded. In this context, periodic multimedia data transmissions are unnecessary, since in such case the camera node will transmit almost the same content, which causes an ineffective expenditure of energy. Thus, the camera nodes should trigger the multimedia transmission only in case of special events based on physical scalar sensor data measurements collected by the scalar sensor nodes. 
The physical scalar sensor measurements are processed using existing models or methods, intending to predict the occurrence of events, such as flooding, fire or intruders. On the other hand, the delivered video will be useful to provide more precise information about the monitored environment than simple scalar data does. Thus, the multimedia data will enable the end-user (or system) to visually identify the real impact of the event, take consciousness of what is happening in the environment, plan actions and help to detect objects, intruders or to analyze scenes. Meanwhile, the routing protocol must assure multimedia delivery with a minimum video quality level from the user perspective to enable carrying out these tasks.

As expected in such scenarios, the locations of camera nodes are pre-defined by the network administrator, according to the physical characteristics of the environment and local policies. On the other hand, the scalar sensor nodes can be deployed in a uniform or random way, e.g., depending on the topology, application requirements or cost issues.

Similar to other hierarchical routing protocols, MEVI considers that $\mathrm{CHs}$ should be used for routing, slot allocation, synchronizing non- $\mathrm{CH}$ transmissions, multimedia retrieval and data aggregation. Thus, a $\mathrm{CH}$ should be a powerful node, and MEVI assumes that camera nodes act as CHs. On the other hand, non-CHs are used for simple tasks, such as detecting scalar physical measurements, and the scalar sensor node acts as a non- $\mathrm{CH}$. In view of the low cost of the network, the number of camera nodes should be as few as possible.

Data transmission consists of two phases: (i) between the non- $\mathrm{CH}$ and its $\mathrm{CH}$ (called intra-cluster communication); and (ii) between $\mathrm{CHs}$, and between $\mathrm{CH}$ and BS (called intercluster communication). The intra and inter-cluster communication comprises a super-frame, as illustrated in Figure 2.

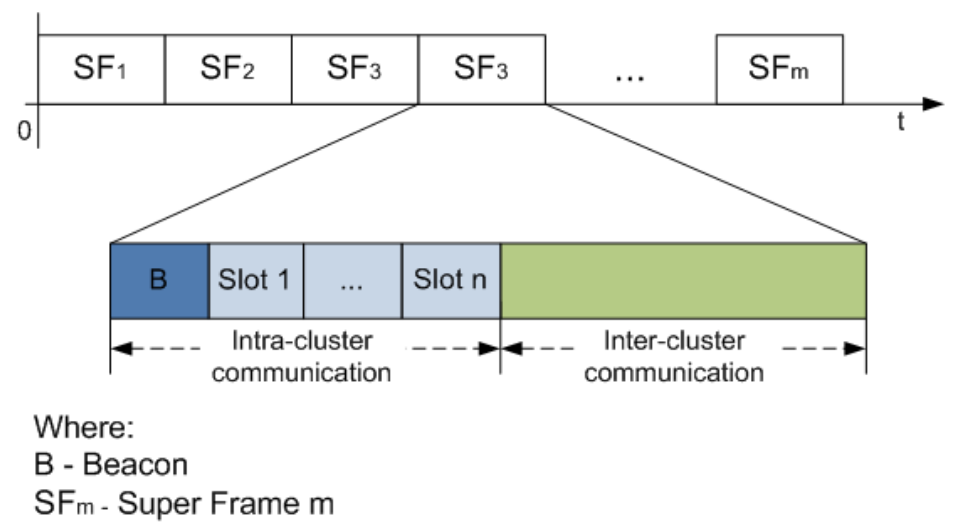

Figure 2 - Super-frame structure

MEVI contains a set of parameters for intra and inter-cluster communication: (i) timeslot duration $\left(\mathrm{t}_{\text {slot }}\right)$ indicates the time interval that a node can take to transmit its packets, (ii) super-frame size $(n)$ indicates the number of contained time-slots; and (iii) the total amount of time for intra and inter-cluster communication is denoted as Round (R).

\subsection{Intra-Cluster Communication}

During this phase, the nodes create clusters and the non-CHs send the sensed values to 
their $\mathrm{CH}$ during their time-slot. An overview and a set of activities of intra-cluster communication process are shown in Figure 3.

The non-CHs remain in sleep mode until the beginning of a new super-frame (Step 1), started by a beacon message, which is sent by the CHs (Step 2). MEVI considers that the beacon message contains a slot map, reporting which slots are idle or busy. Compared with other hierarchical routing protocols, such as LEACH and PEMuR, the beacon message in MEVI is a combination of schedule and beacon messages.

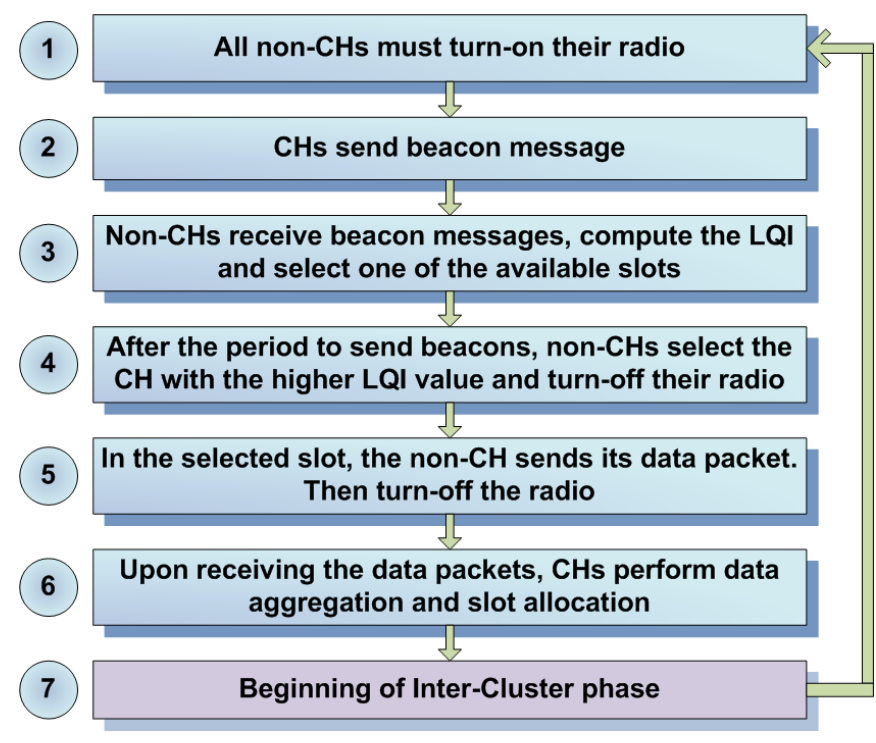

Figure 3 - Set of activities for cluster formation

MEVI relies on the Link Quality Indicator (LQI) as the metric to a select a reliable $\mathrm{CH}$. LQI is provided by the physical layer of IEEE 802.15.4 and can be used to improve reliability of the system. The LQI is computed at each received beacon message and MEVI takes into account the degree of variability of the links to select the $\mathrm{CH}$. Specifically, it computes the weighted arithmetic average from the last $x$ LQI values so that it can give more importance to the recent LQI values (Step 3).

In contrast to related work on hierarchical routing protocols, where non-CHs send a join message for a $\mathrm{CH}$, the $\mathrm{CH}$ chooses a time-slot for each non- $\mathrm{CH}$ and then sends a schedule message. In MEVI, the non-CHs have to become aware of which time-slots are idle or busy, by analyzing the slot map contained in a beacon message (Step 3). After the period when the $\mathrm{CHs}$ send beacon messages, a non- $\mathrm{CH}$ should select the $\mathrm{CH}$ with the highest LQI average, which means that the most reliable $\mathrm{CH}$ is selected to receive the non- $\mathrm{CH}$ scalar sensor data packets (Step 4). Following this, the non-CH waits for its selected time-slot, and sends the sensed physical data (Step 5).

In this paper, we propose a cluster formation scheme with a low overhead. Where, beacon messages include the TDMA schedule, and data packets include a join message. Thus, before the non-CHs allocate a time-slot, they have to wait for a beacon message and then send their data packet during the selected time-slot. Hence, it is different from existing hierarchical routing protocols presented in Section 2, where the nodes at least have to 
exchange beacon, join and schedule messages, before sending their data packets. Therefore, with the reduced signaling overhead in MEVI, it is possible to increase network lifetime.

The $\mathrm{CH}$ receives the data packets and assigns a time-slot according to the following rule (Step 6): if only one node tries to allocate the slot, the slot will be successfully allocated; otherwise, the $\mathrm{CH}$ will assign the slot at random to one of the candidate nodes. After finishing the $n$ time-slots, the inter-cluster phase starts (Step 7), which is explained in Section 3.2.

The non-CHs should wait for the next beacon so that they can recognize a valid transmission in the selected slot. If this holds, it means that the selected slot was successfully allocated, and the non- $\mathrm{CH}$ must keep sending the sensed data in the same slot. Otherwise, the non- $\mathrm{CH}$ must repeat the procedure until a slot assignment is obtained.

It is important to highlight that the non-CHs only turn on their radio in the period when the $\mathrm{CHs}$ send beacons and in their own time-slot. If the $\mathrm{CH}$ detects that a slot has not been used for the last $y$ super-frames (called the idleness of a time-slot), it will be considered idle in the next beacon message. After the non- $\mathrm{CH}$ has allocated a time-slot, it should switch to another $\mathrm{CH}$ only if it detects that the LQI average of another $\mathrm{CH}$ candidate is higher than the current one.

\subsection{Inter-Cluster Communication}

The inter-cluster communication is the period when $\mathrm{CHs}$ and the $\mathrm{BS}$ are communicating with each other. This period is used by the $\mathrm{CHs}$ to send the aggregate and multimedia data packets to the BS, and the BS can request multimedia content for a $\mathrm{CH}$ if necessary. In case of an event, upon receiving scalar or multimedia data, the users, authorities, or systems are able to decide a suitable action with more precise information.

MEVI uses the sensed scalar physical measurements, e.g. temperature, humidity and others, to take appropriate decisions with regard to multimedia retrieval and transmission. Basically, if one of the sensed values is higher than a soft or hard threshold, the camera nodes should select different actions for multimedia retrieval. These actions are named of operational modes, and can be normal and event mode. The periods that comprise these modes are illustrated in Figure 4.

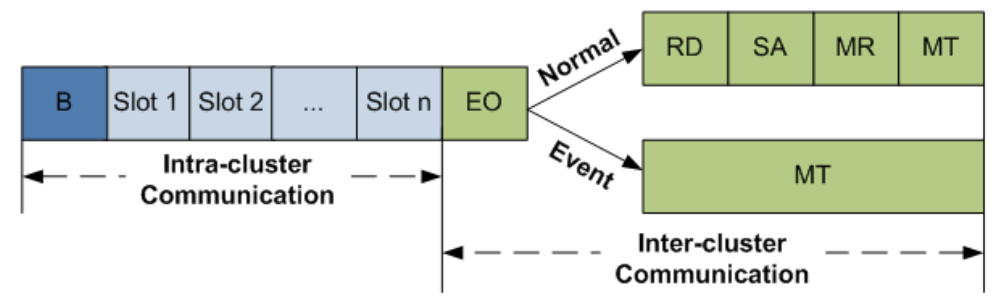

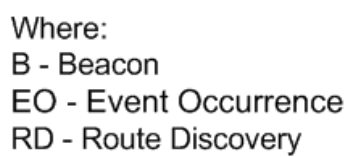

Where:

EO - Event Occurrence

RD - Route Discovery
SA - Send Aggregate Packet

MR - Multimedia Request

MT - Multimedia Transmission

Figure 4 - Inter-Cluster Communication Structure 
In normal mode, $\mathrm{CHs}$ are not continuously sending video, with the aim of saving energy and extending network lifetime. For this mode, non-CHs are continuously sending the sensed physical environmental conditions to the selected $\mathrm{CH}$, which aggregates the non-CHs data into a single packet and sends it to the BS. Video content is requested by the BS, if one of the sensed values is higher than a soft threshold, e.g. for a fire detection application, temperature higher than $50^{\circ} \mathrm{C}$ means that there is a possibility of an event occurrence.

Specifically, if the CHs do not receive any event message during the Event Occurrence period (EO), it means that the nodes should remain in normal mode, which works as follows. Route Discovery (RD) is the period when $\mathrm{CHs}$ find routes to the BS. MEVI exploits a reactive scheme to find routes on demand, with the aim to decrease the overhead and to improve scalability of the system. RD uses pairs of route request (RREQ) and reply (RREP) messages. It is important to highlight that the transmission of $R R E Q$ and $R R E P$ messages in hierarchical networks is different from flat network. Due to the fact that only CHs need to find routes to $\mathrm{BS}$, only $\mathrm{CHs}$ have to keep the radio turned on during $\mathrm{RD}$. Each path has associated a Link Quality (LQ) value, which is used to score and classify the links. The LQ is computed based on cross-layer information according to (1). The equation considers Remaining Energy (RE), LQI, Hop Count (HC) and weights to give a degree of importance to each metric.

$$
L Q=\alpha \times \frac{R E}{E_{0}}+\beta \times \frac{L Q I}{\max L Q I}+\gamma \times \frac{\max H C-H C}{\max H C}
$$

where, $0 \leq \mathrm{LQ} \leq 1, \alpha+\beta+\gamma=1$, E0 is the initial energy of a node, maxLQI $=255$, and maxHC depends on the network diameter.

The path with the highest LQ has better conditions to transmit packets, which means there is lower packet loss. However, links change over time due to network conditions, and it is desirable to periodically update the LQ. This is the reason why every normal mode includes a RD period. To compute the LQ, the CHs should know the RE and HC values of each neighbor $\mathrm{CH}$. Thus, RREQ and RREP messages have to include additional fields to report these values.

Once the $\mathrm{CHs}$ have routes, they are able to transmit their aggregate packet to the BS during the Send Aggregate packets (SA) period. As soon as the BS receives the aggregate packets, it will analyze the data. If the recent history of the non-CHs sensed scalar data is higher than a soft threshold, e.g., temperature higher than $50^{\circ} \mathrm{C}$ for fire detection applications, this can be a possibility of an event occurrence, and multimedia data is used to verify event occurrence and visually identify the real impact of the incident, e.g., fire, in the environment. In this case, the $\mathrm{BS}$ has to request multimedia content from a $\mathrm{CH}$ and to trigger route discovery if it does not have a route to the $\mathrm{CH}$, during the Multimedia Request (MR) period. When the $\mathrm{CH}$ receives the multimedia request message, it will turn the FoV to the location of the non- $\mathrm{CH}$ where there is the possibility of an event occurrence, as shown in Figure 5. Then, it retrieves and transmits video content. 
Scalar sensor nodes, i.e. non-CHs, have a sensor range of a disk, which means that they can sense scalar physical measurements from all directions, i.e., in an omnidirectional way. On the other hand, the sensing range of a camera node, i.e. CHs, is called Field-of-View (FoV). FoV is defined as a triangle, which depends on the direction of the camera (V), angle of view $(\alpha)$ and a depth of view (d), as shown in Figure 5. Thus, the sensing range of a camera node is limited, and depends on the direction of the camera and its features for angle and depth of view.

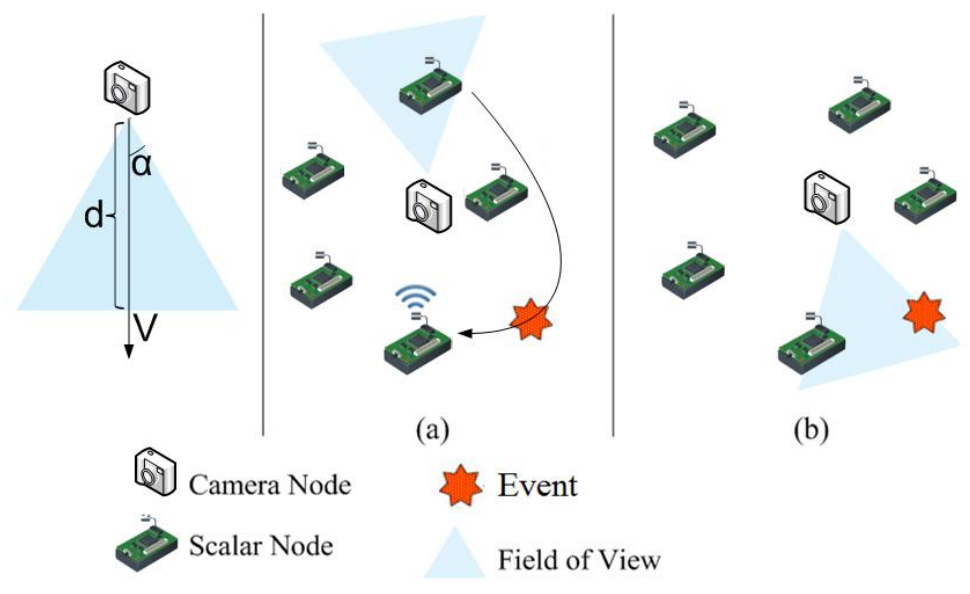

Figure 5 - Tuneable Field of View

To enable the camera node to change the direction of its FoV to the event area, some initialization work is needed. In the first super-frame, the scalar sensor nodes should broadcast their location information to neighborhood nodes. The camera node receives and stores this information. Then, when the camera node has to send multimedia content, it is possible to change the direction of its FoV to the location of the scalar sensor node where there is an event occurrence.

On the other hand, if the $\mathrm{CH}$ detects that one of the non-CHs sensed values is higher than a hard threshold (e.g., temperature higher than $90{ }^{\circ} \mathrm{C}$ for a fire detection application), this means that an event has already happened and the nodes must provide as much visual information as possible to allow the system administrator to visually identify the real impact of the event. In this case, CHs should start the event mode and do not need to save energy resources. This is due to the fact that there is an event occurrence, and the nodes could be destroyed, e.g. by a fire. As mentioned before, multimedia content provides more precise information than simple scalar data, enabling the end-user to visually verify the real impact of the event, take consciousness of what is happening in the environment, to plan actions according to these visual information and to help detecting objects or intruders or/and analyzing scenes.

The $\mathrm{CH}$ should start event mode by sending an event message through a path used to reach the BS in an Event Occurrence (EO) period. The CHs that compose a path to reach the BS should forward the message with the aim of informing the nodes about the event occurrence mode. In this way, it is possible to avoid interference and give priority to multimedia transmission from the site of the event. 
After CHs became aware of the event mode, the multimedia transmission (MT) period has to start. The $\mathrm{CH}$ will retrieve and send the multimedia content to the $\mathrm{BS}$ using multiple hops, which have been found previously in normal mode.

\section{Performance Evaluation}

\subsection{Simulation Scenario}

Simulation experiments were conducted to analyze the performance of MEVI by using the extended version of Wireless Simulation Environment for Multimedia Networks (WiSEMNet) [13], which is based on OMNeT++ [14]. The extended version of WiSE-Mnet [13], integrates the functionalities of WiSE-Mnet [15], Wvsnmodel [16] and Evalvid [17]. Also, it implements the change of the direction of the camera's FoV to retrieve multimedia content from the target area.

WiSE-MNet incorporates some functionalities from Castalia, and it provides a generic network-oriented simulation environment that addresses the need for co-design of network protocols and distributed algorithms for WMSNs. Wvsnmodel efficiently defines a model to find subsets of nodes to cover a given area (denoted as cover-set), and defines the sensing range by a FoV and not by a disk. Evalvid provides support for the transmission, control and evaluation of real video sequences in simulation environments.

Simulations were carried out and repeated 20 times to provide a confidence interval of 95\% to show the impact and benefits of MEVI proposed in this paper for dissemination of video content in WMSNs. The performance of MEVI was compared with LEACH, PEMuR and MEVI variations in small (scenario 1) and large scale (scenario 2) scenarios. Table 2 shows the simulation parameters used for the simulations.

For this paper, the Container video sequence was chosen from the Video Trace Library [19]. The video uses the QCIF format, since this is more suitable for WMSNs, as shown in [20]. The authors usually classify the videos according to their motion and complexity into three categories, namely low, median and high complexity. According to [21], the Container video sequence is classified as low movement, which means that it has a small moving region of interest on a static background, i.e. a ship crossing a lake. This video characteristic is expected for many WMSNs applications, such as environmental monitoring and smart parking.

Traditionally, routing protocols were evaluated from network/packet level point-of-view by using Quality of Service (QoS) metrics, e.g., delay, jitter, or loss. However, QoS metrics do not reflect the user's perception and, consequently, fail in capturing subjective aspects associated with human experience. Quality of Experience (QoE) metrics/approaches overcome the limitations of current QoS-aware routing schemes regarding to human perception and subjective-related aspects [3] [6]. Therefore, to highlight the MEVI reliability from the user point-of-view, the simulation evaluates MEVI by using mainly QoE metrics.

The quality of the transmitted video was evaluated by using well-known objective QoE 


\section{Macrothink}

metrics, i.e. Structural Similarity (SSIM) and Video Quality Metric (VQM). SSIM is a measurement of the structural distortion of the video, which tries to obtain a better correlation with the user's subjective impression. SSIM has values ranging from 0 to 1 , a higher value means better video quality. The VQM metric measures the "perception damage" the video experienced, based on features of the human visual system, including distinct metric factors such as blurring, noise, color distortion and distortion blocks. A value closer to 0 means a video with a better quality.

Table 2 - Simulation Parameters

\begin{tabular}{|c|c|c|}
\hline Parameters & Scenario 1 & Scenario 2 \\
\hline Field size & $40 \times 40$ & $80 x 80$ \\
\hline Location of BS & $(20,40)$ & $(40,80)$ \\
\hline Transmission power for multi-hop protocols & $-10 \mathrm{dbm}$ & $-3 \mathrm{dbm}$ \\
\hline Transmission power for single-hop protocols & $-1 \mathrm{dbm}$ & 0 dbm \\
\hline Total number of nodes & \multicolumn{2}{|c|}{100} \\
\hline Number of camera nodes & \multicolumn{2}{|c|}{25} \\
\hline Initial energy $\left(\mathbf{E}_{0}\right)$ & \multicolumn{2}{|c|}{$10 \mathrm{~J}$} \\
\hline Soft threshold & \multicolumn{2}{|c|}{$50{ }^{\circ} \mathbf{C}$} \\
\hline Hard threshold & \multicolumn{2}{|c|}{$90{ }^{\circ} \mathbf{C}$} \\
\hline Number of slots $(n)$ & \multicolumn{2}{|c|}{10} \\
\hline Duration of each slot $\left(t_{\text {slot }}\right)$ & \multicolumn{2}{|c|}{1 second } \\
\hline Round duration (R) & \multicolumn{2}{|c|}{30 seconds } \\
\hline Maximum number of hops & \multicolumn{2}{|c|}{8} \\
\hline Radio propagation model & \multicolumn{2}{|c|}{ Lognormal shadowing model } \\
\hline Radio & \multicolumn{2}{|c|}{$\mathrm{CC2420}$} \\
\hline Video sequence & \multicolumn{2}{|c|}{ Container } \\
\hline Video encoding & \multicolumn{2}{|c|}{ MPEG-4 } \\
\hline Video format & \multicolumn{2}{|c|}{ QCIF $(176 \times 144)$} \\
\hline
\end{tabular}

SSIM and VQM values are obtained by using the MSU Video Quality Measurement Tool (VQMT). During the simulations, CHs transmitted several times the Container video sequence, and the video quality metrics presented here are the average of SSIM or VQM for all transmitted Container video sequence of each $\mathrm{CH}$.

An analysis was conducted using the nodes that were still alive after some rounds (network lifetime). The network lifetime was measured as the period of time until $10 \%$ of the nodes have run out of energy.

\subsection{Performance Evaluation}

Simulations were conducted to study the impact and benefits of MEVI in disseminating video content in WMSNs, for large and small-scale scenarios (denoted as MEVI in the 


\section{Macrothink Institute}

results). Following this, two simple versions of MEVI was simulated by using single-hop communication between the CHs and BS (denoted as MEVI single-hop in the results). MEVI single-hop is used to show the advantages of using multiple hops. Additionally, we simulated another version of MEVI using multiple hops, by scoring and selecting the routes on the basis of a hop count (denoted as MEVI Hop-count in the results). MEVI Hop-count is useful to presents the benefits of using the proposed cross-layer mechanism to select routes based on network conditions and energy issues.

MEVI was also compared with two well-known related works on hierarchical routing protocols, namely LEACH and PEMuR. We implemented two versions of LEACH. The first version takes into account the traditional implementation of LEACH, by including multimedia transmission after the time-slots period. The second LEACH version (LEACH Fixed $\mathrm{CH}$ ) has the same features of MEVI in terms of heterogeneous node capabilities, i.e. it considers that the $\mathrm{CHs}$ are more powerful node. $\mathrm{LEACH}$ Fixed $\mathrm{CH}$ has the same process for cluster formation as the original LEACH version, i.e. using beacon, join and schedule message, and can be used to evaluate the benefits of our proposed cluster formation scheme with reduced signaling overhead.

Figure 6 shows the number of nodes per round that were still alive for small (scenario 1) and large scale (scenario 2) scenarios, the scenarios characteristics was described in Table 1. We only examine the results for one version of MEVI. The main reason is due to the fact that $\mathrm{CHs}$ have a rich energy source. Additionally, non-CHs never become $\mathrm{CHs}$, and thus they only turn on the radio during the beacon period to send the data packets. Thus, the number of nodes alive per round is the same for all the MEVI versions.

PEMuR and LEACH consider nodes with homogeneous capabilities and in each round, a different set of nodes is elected to serve as CHs. In the hierarchical architecture, the CHs consume more energy to transmit or forward packets to the BS. Basically, this explains the higher gain in terms of the network lifetime by MEVI.

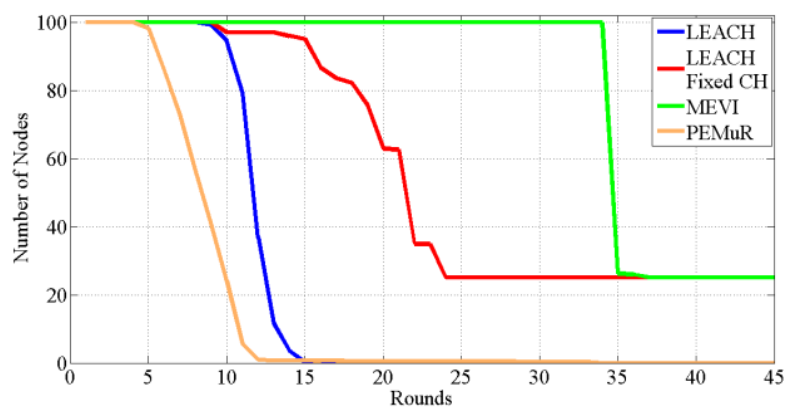

(a) Nodes that still alive for Scenario 1

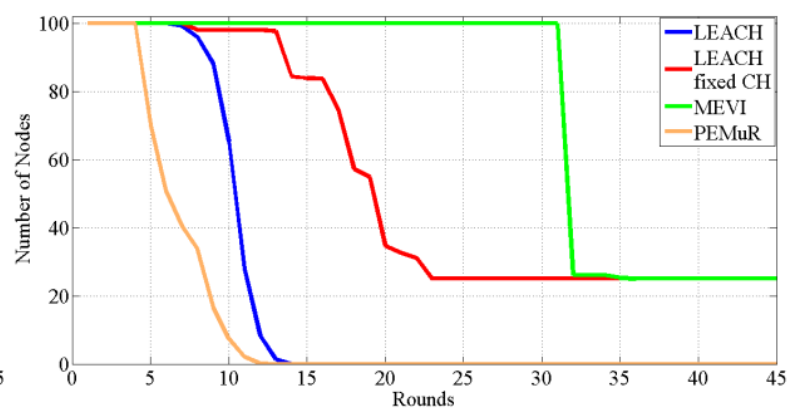

(b) Nodes that still alive for Scenario 2

Figure 6 - Average of Network Lifetime

When MEVI and LEACH are compared for scenarios 1 and 2, MEVI increased the network lifetime by 70\%, (Figure 5 (a) and (b), respectively). The reason for this is that the $\mathrm{CHs}$ in LEACH have to use higher transmission power to send packets to the BS, i.e. -1 or 0 $\mathrm{dbm}$ depending on the scenario, in contrast with MEVI which uses -10 or $-3 \mathrm{dbm}$. Higher 
transmission power means higher energy consumption.

PEMuR has the worst network lifetime, i.e. around 10 rounds, due to the fact that PEMuR has more control messages for cluster formation compared with original LEACH. Additionally, PEMuR is a multi-hop protocol and the $\mathrm{CHs}$ have to forward a higher number of multimedia packets, which consumes more energy compared to LEACH. Finally, when MEVI and LEACH Fixed CH are compared, MEVI increases network lifetime by 50\%. This is because MEVI reduces signaling overhead for cluster formation to a lower degree compared to LEACH.

The video quality varies depending on the distance of the camera node to the BS. A further distant node suffers higher packet loss, due to the fact that more hops are needed to reach the BS, which increases interference and drops on restricted buffers. This is because video content creates a huge amount of data that has to be transmitted. Thus, in this study, our analysis of video quality is in accordance with the distance from the camera node to the BS. It is important to highlight that although SSIM and VQM range from 0 to 1 and 0 to 5, respectivelly. We establish an interval from 0.4 to 1 for SSIM, and from 0 to 3 for VQM to enlarge the differentiation between protocols.

First, we analyze the video quality of nodes with distances from $0 \mathrm{~m}$ to $11 \mathrm{~m}$, for a smallscale scenario, i.e. for scenario 1; see Figure 7 and Figure 8. For nodes near the BS the videos have a similar quality regardless of the protocols. The reason for this is that multi-hop protocols need a few hops to reach the BS, i.e., 1 or 2 hops. On the other hand, for single-hop protocols, the camera nodes are close to the BS and use higher transmission power. Thus, there are less packet losses, which improve the video quality.

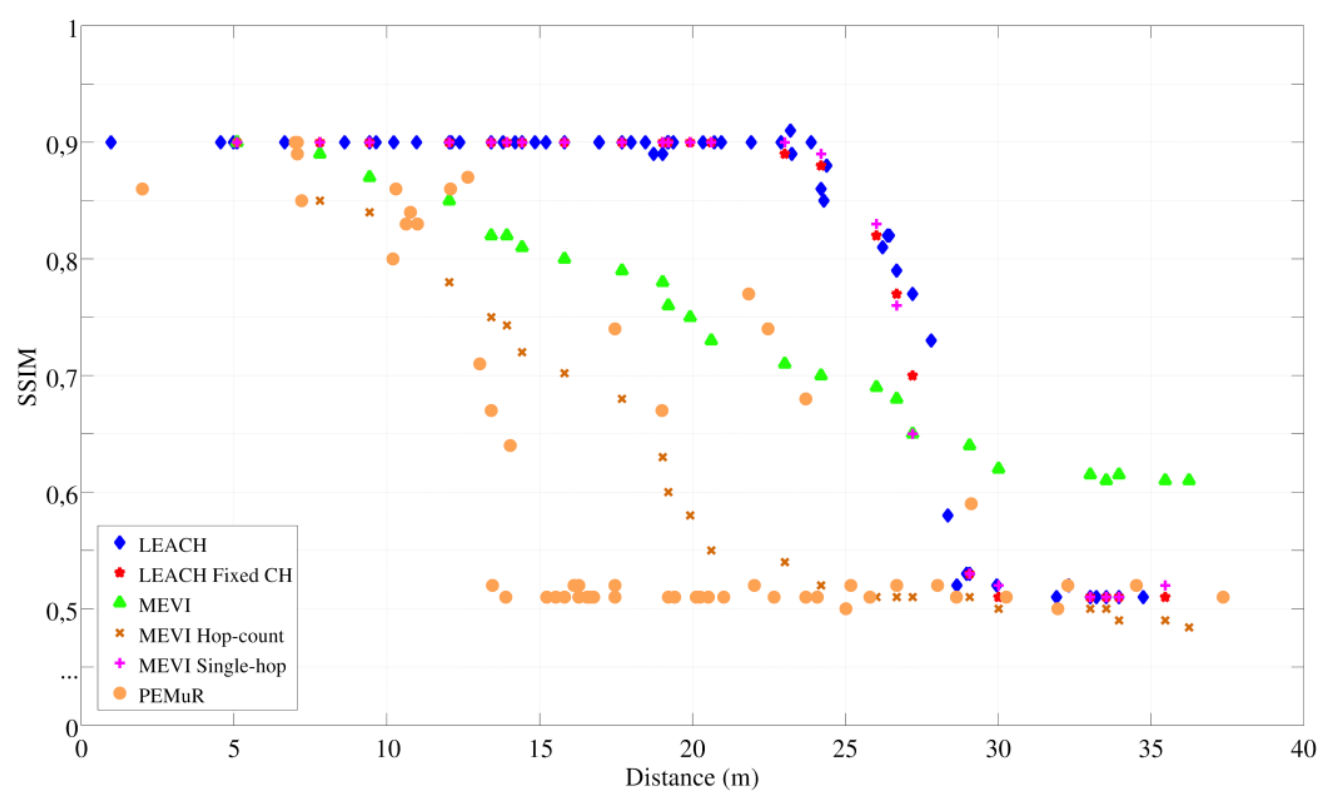

Figure 7 - SSIM according to distance from the camera node to BS for Scenario 1

Analyzing the video quality for distances between $11 \mathrm{~m}$ and $26 \mathrm{~m}$ for scenario 1 , singlehop protocols still have videos with higher quality, i.e. 0.9 for SSIM and 0.5 for VQM, compared with multi-hop protocols, which range from 0.9 to 0.5 for SSIM and 0.5 to 2.5 for 


\section{Macrothink}

VQM. The reason for this behavior is that for single-hop protocols the camera nodes send multimedia packets using higher transmission power, and the BS is still in the transmission range of such nodes to receive packets with higher reliability. On the other hand, multi-hop protocols use lower transmission power through multiple hops, which causes more packet loss compared to single-hop protocols. Analyzing the multiple hops protocols, PEMuR has the worst performance, because it selects routes based only on remaining energy, which is not an appropriate metric to select reliable routes.

Nevertheless, for distances above $26 \mathrm{~m}$ for scenario 1 , MEVI increases the video quality by $20 \%$ for SSIM and $60 \%$ for VQM, compared with other solutions. This improvement is due to the fact that MEVI uses multiple hops, with a cross-layer solution to select reliable routes based on network conditions, i.e. LQI, remaining energy, and number of hops. On the other hand, for single-hop protocols, i.e. LEACH, LEACH Fixed CH and MEVI Single-hop, the camera nodes are not able to deliver packets to the BS with higher reliability, even using higher transmission power. PEMuR and MEVI Hop-count select routes based on remaining energy or number of routes respectively. This makes such proposals unreliable, because they do not consider cross-layer information to select routes. This causes more packet loss and lowers video quality.

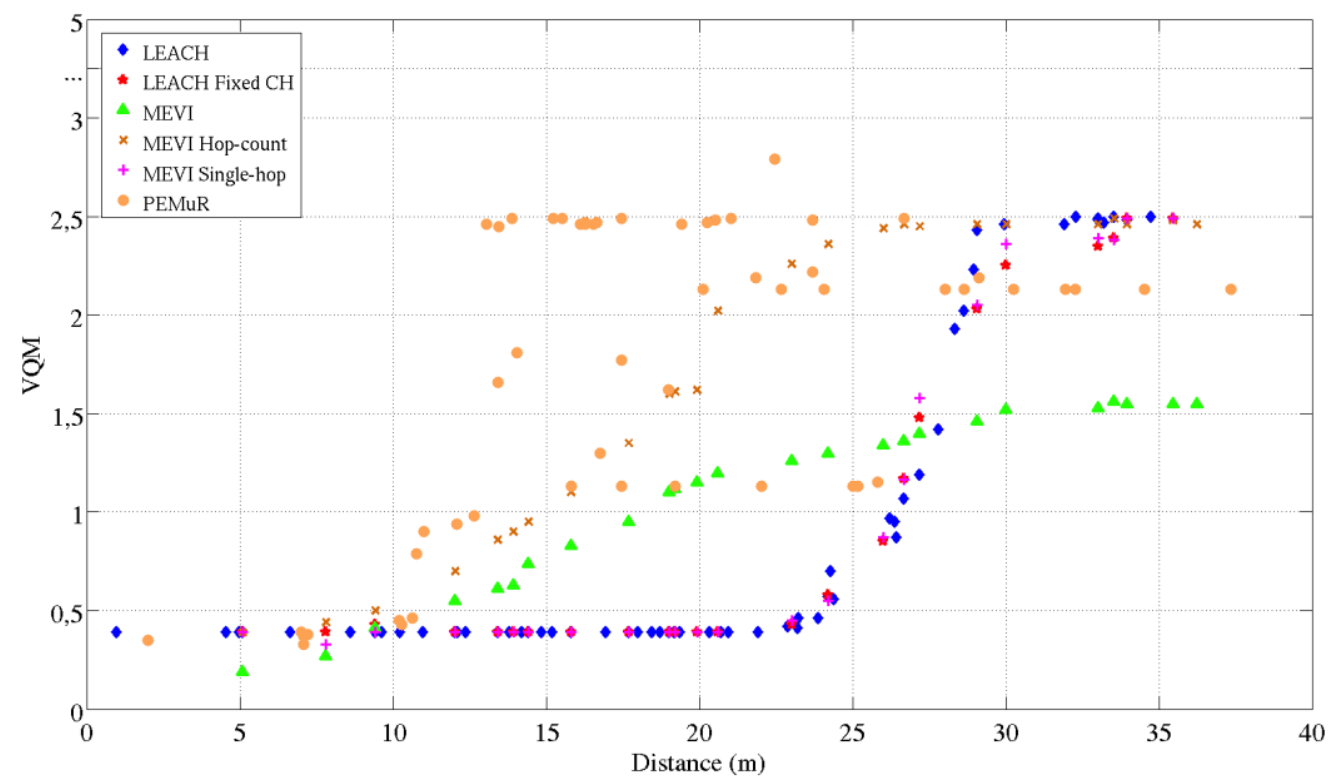

Figure 8 - VQM according to distance from the camera node to BS for Scenario 1

As mentioned earlier, MEVI was also evaluated in large-scale scenarios, as expected in smart cities and environmental monitoring applications. In this way, it is possible to analyze if MEVI can still deliver video with good quality regardless of distance. The SSIM and VQM of nodes with distances from $0 \mathrm{~m}$ to $20 \mathrm{~m}$ were analyzed for a large-scale scenario, i.e. scenario 2, see Figure 9 and Figure 10. The delivered videos have a similar video quality regardless of the protocols; the reasons for this are the same as for scenario 1. 


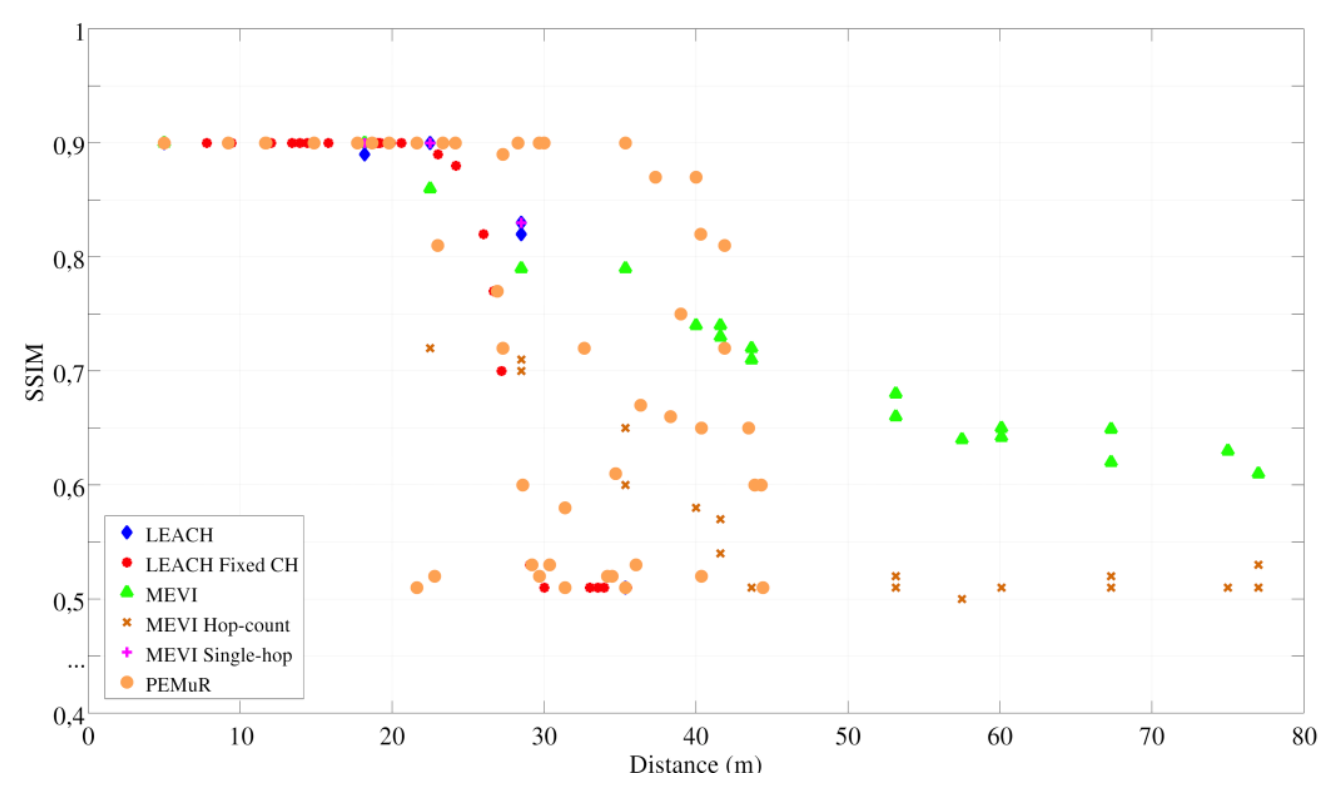

Figure 9 - SSIM according to distance from the camera node to BS for Scenario 2

For distances longer than 40m, PEMuR and single-hop protocols, i.e. LEACH, LEACH Fixed $\mathrm{CH}$ and MEVI Single-hop, the BS is not within the transmission rage of $\mathrm{CHs}$, and thus such protocols are not able to send multimedia packets to the BS with better reliability. On the other hand, MEVI and MEVI Hop-count still deliver videos to the BS, due to the use of multiple hops. However, MEVI increases the video quality by $20 \%$ for SSIM and VQM compared to MEVI Hop-count. This improvement of MEVI is because the uses of multiple hops with a cross-layer solution to select reliable routes, which decreases the packet loss and increase the video quality. The worst performance of MEVI Hop-count compared to MEVI is due to the fact that routes are selected based on the number of hops, which is not an appropriate metric to select reliable routes.

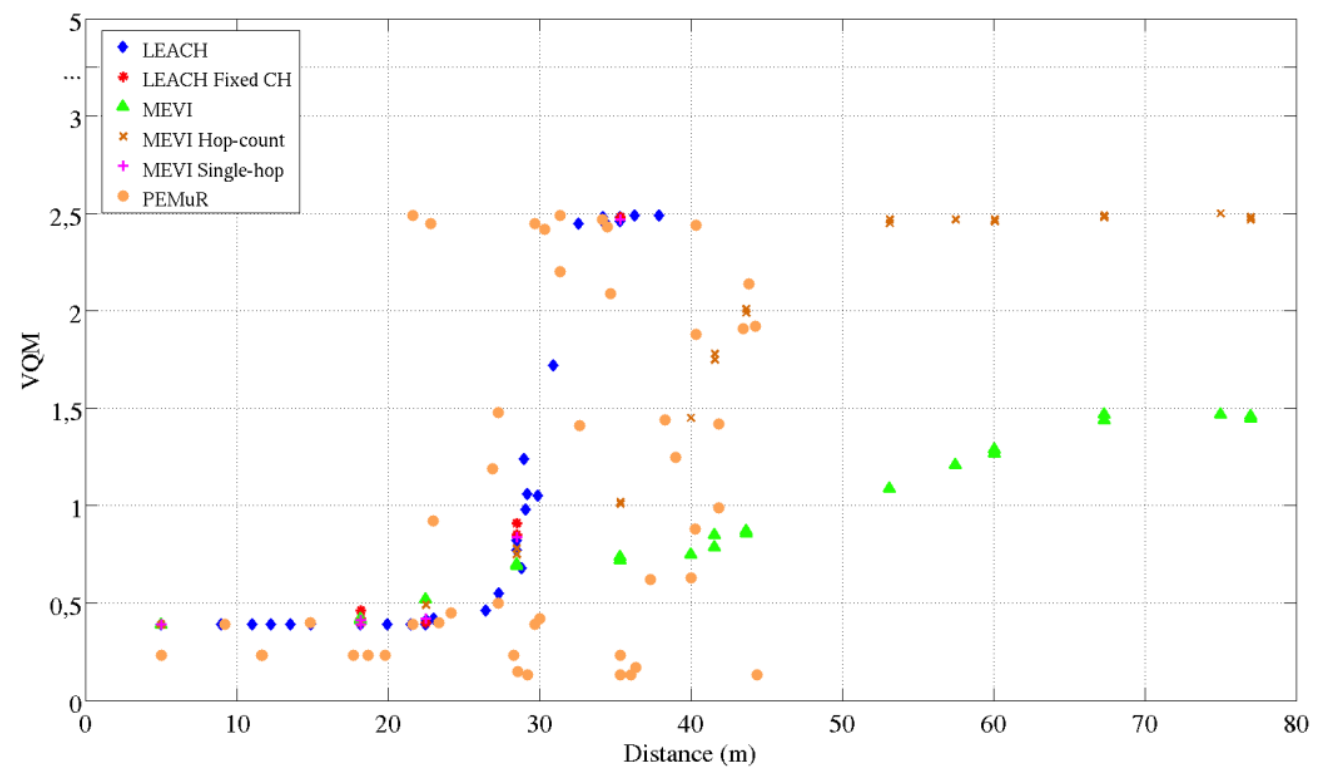

Figure 10 - VQM according to distance from the camera node to BS for Scenario 2 


\section{Macrothink Institute ${ }^{\mathrm{TM}}$}

To show the impact of transmitting video streams using MEVI from the standpoint of the end-user, a random frame was selected (frame 266) from the transmitted video, as displayed in Figure 11. The benefits of MEVI are evident when compared with other protocols using the original and the received frame. Frame 266 is the moment when a bird appears and flies across the scene. This is the interval of the video with higher mobility. Thus, in some applications, the moment of a video with higher mobility is useful to provide more precise information, enabling the end user (or system) to visually verify the real impact of the event, take consciousness of what is happening in the environment, to plan actions according to these visual information and to help detecting objects or intruders or/and analyzing scenes.

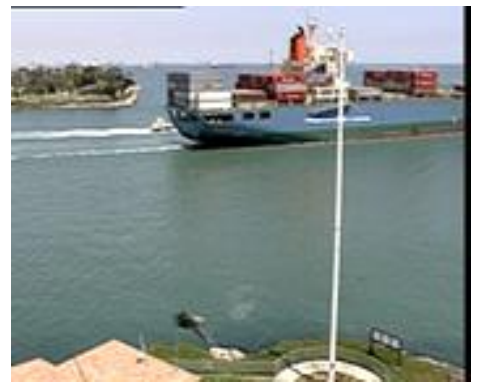

(a) Original Frame

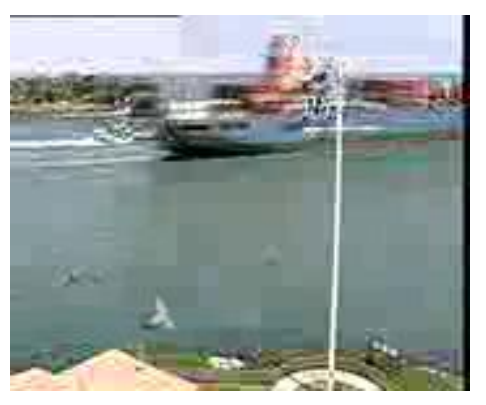

(d) MEVI Hop-count

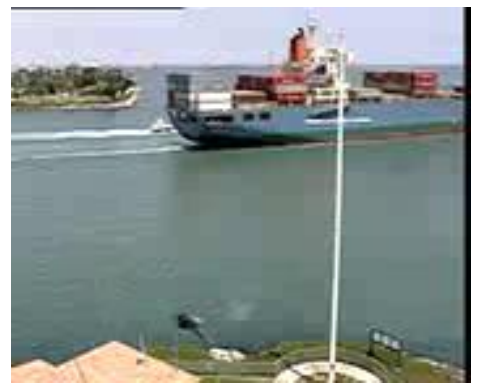

(b) MEVI

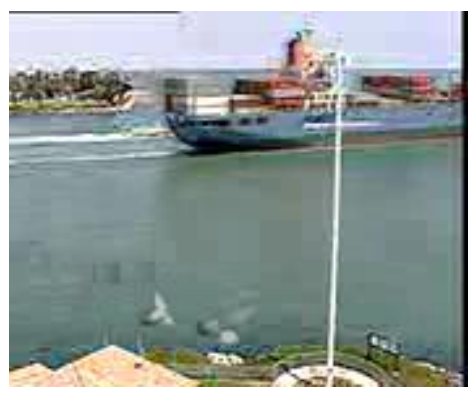

(e) LEACH

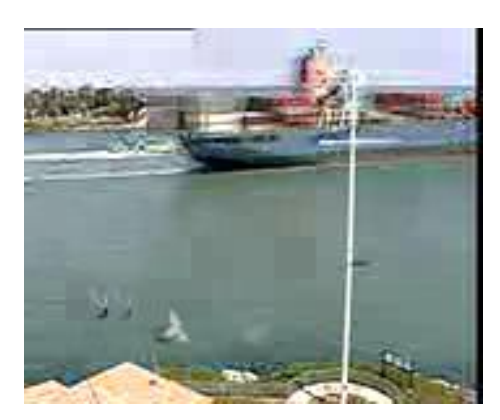

(g) PEMuR

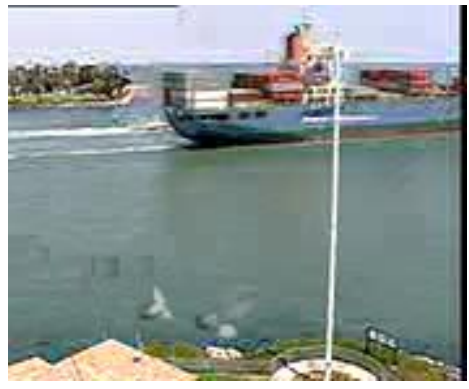

(c) MEVI Single-hop

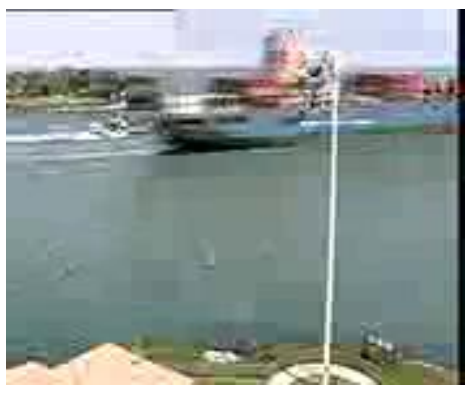

(f) LEACH Fixed-CH

(g) PEMuR

Figure 11 - Frame 266 transmitted with different routing protocols

When analyzing Figure 11, it is possible to visually observe that MEVI allows video transmission with excellent quality from the user perspective. This can be concluded from Figure 10b, where for the transmission of frame 266, MEVI has only a few distortions and the bird appears to be in the same position, compared with the original frame. 
On the other hand, the transmission of frame 266 using MEVI Single-hop, MEVI Hopcount, LEACH, LEACH Fixed-CH and PEMuR has low video quality from the user perspective, as shown in Figure 11 (c), (d), (e), (f), and (g) respectively. For these protocols, the bird does not appear in the same position. Moreover, there is a higher distortion in the ship and the lake compared with the original frame. The reason is that MEVI Single-hop, MEVI Hop-count, LEACH, LEACH Fixed-CH and PEMuR are not reliable enough to send multimedia packets as explained in this section. Thus, there is a higher packet loss, since route selection does not take cross-layer information into account or use single hop communication. The reason for the bird not appearing at the same position compared to the original frame is that this frame was lost, and thus this frame was reconstructed on the basis of the previously received frames.

\section{Conclusion}

This paper has outlined a smart Multi-hop hierarchical routing protocol for Efficient VIdeo communication over WMSNs (MEVI). MEVI combines a cluster formation scheme with low overhead, and ensure reliable multi-hop communication between $\mathrm{CHs}$ and BS. For route selection, a cross-layer solution based on network conditions, energy and number of hops was used. Additionally, the nodes retrieve and send multimedia content in accordance with the sensed physical environmental conditions.

Simulations were carried out to show the benefits of MEVI in disseminating multimedia content for large and small field size. From the results of the simulations, it was found that MEVI increases network lifetime by at least $60 \%$ for small and large scale scenarios compared with LEACH, PEMuR and simple versions of MEVI. The video quality for MEVI transmissions is increased by at least $20 \%$ for small field sizes. Nevertheless, for large field sizes, MEVI still able to deliver video, unlike the related protocols that are not able to send video content on a large scale.

\section{Acknowledgement}

This work was supported by the National Council for Scientific and Technological Development (CNPq), CNPq Scholarship and the Foundation for Research of the State of Para (FAPESPA) - Brazil as well as the Swiss nano-tera research program by funding the SSSTC research project Mobile Multi-Media Wireless Sensor Networks (M3WSN).

\section{References}

[1] Yick J., Mukherjee B., Ghosal D., "Wireless sensor network survey," Computer networks, vol. 52, no. 12, pp. 2008. http://dx.doi.org/10.1016/j.comnet.2008.04.002

[2] Almalkawi I., Guerrero Zapata M., Al-Karaki J., Morillo-Pozo J., "Wireless Multimedia 
Sensor Networks: Current Trends and Future Directions," Sensors, vol. 10, no. 7, pp. 66626717, 2010. http://dx.doi.org/10.3390/s100706662

[3] Rosário D., Machado K., Abelém A., Monteiro D., and Cerqueira E., "Recent Advances and Challenges in Wireless Multimedia Sensor Networks", Mobile Multimedia - User and Technology Perspectives, Dian Tjondronegoro (Ed.), ISBN: 978-953-307-908-0, InTech, pp. 74-96, 2012. http://dx.doi.org/10.5772/27712

[4] Ehsan S., Hamdaoui B., "A survey on energy-efficient routing techniques with QoS assurances for wireless multimedia sensor networks," IEEE Communications Surveys $\begin{array}{llllllll}\text { Tutorials, } & \text { vol. } \quad 14, \quad \text { no. } \quad 99, \quad \text { pp. } & 265 & - & 278, & 2012 .\end{array}$ http://dx.doi.org/10.1109/SURV.2011.020211.00058

[5] Rosário D., Costa R., Paraense H., Machado K., Cerqueira E. and Braun T., "A smart multi-hop hierarchical routing protocol for efficient video communication over wireless multimedia sensor network," in 2nd IEEE International Workshop on Smart Communication Protocols and Algorithms (ICC'12 WS - SCPA), Ottawa, Canada, Jun. 2012. http://dx.doi.org/10.1109/ICC.2012.6364926

[6] Serral-Gracia R., Cerqueira E., Curado M., Yannuzzi M., Monteiro E. and Masip-Bruin $\mathrm{X}$., "An overview of quality of experience measurement challenges for video applications in IP networks," Wired/Wireless Internet Communications, pp. 252-263, 2010. http://dx.doi.org/10.1007/978-3-642-13315-2_21

[7] Heinzelman W., Chandrakasan A. and Balakrishnan H., "Energy-efficient communication protocol for wireless microsensor networks," in 33rd Annual Hawaii International Conference on System 2000. http://dx.doi.org/10.1109/HICSS.2000.926982

[8] Sun Y., Ma H., Liu L. and Zheng Y., "ASAR: An ant-based service-aware routing algorithm for multimedia sensor networks," Frontiers of Electrical and Electronic Engineering in China, vol. 3. http://dx.doi.org/10.1007/s11460-008-0013-7

[9] Cobo L., Quintero A., Pierre S., "Ant-based routing for wireless multimedia sensor networks using multiple QoS metrics," Computer networks, vol. 54, no. 17, pp. 2991-3010, 2010. http://dx.doi.org/10.1016/j.comnet.2010.05.014

[10]Lin K., Chen M., Ge X., "Adaptive reliable routing based on cluster hierarchy for wireless multimedia sensor networks," EURASIP Journal on Wireless Communications and Networking, vol. 2010, p. 3, 2010. http://dx.doi.org/10.1155/2010/567952

[11] Lin K., Rodrigues J., Ge H., Xiong N., Liang X., "Energy efficiency QoS assurance routing in wireless multimedia sensor networks," IEEE Systems Journal, vol. 5, no. 4, pp. 495 -505, dec 2011. http://dx.doi.org/10.1109/JSYST.2011.2165599

[12] Kandris D., Tsagkaropoulos M., Politis I., Tzes A., Kotsopoulos S., "Energy efficient and perceived qos aware video routing over wireless multimedia sensor networks," Ad Hoc Networks, vol. 9, no. 4, pp. 591 - 607, 2011. http://dx.doi.org/10.1016/j.adhoc.2010.09.001 
[13] Zhao Z., Braun T., Rosário D., Cerqueira E., Immich R. and Curado M., "Qoe-aware FEC mechanism for intrusion detection in multitier wireless multimedia sensor networks," in 1st International Workshop on Wireless Multimedia Sensor Networks (WMSN'2012), Barcelona, Spain, Oct. 2012. http://dx.doi.org/10.1109/WiMOB.2012.6379150

[14] Varga A., "The OMNeT++ discrete event simulation system," in Proceedings of the $15^{\text {th }}$ European Simulation Multiconference (ESM 2001), Prague, Czech Republic. June 6-9, 2001.

[15] Nastasi C. and Cavallaro A., "Wise-mnet: an experimental environment for wireless multimedia sensor networks," in Sensor Signal Processing for Defence (SSPD 2011), London, UK, 27-29 Sept. 2011. http://dx.doi.org/10.1049/ic.2011.0171

[16] Pham C. and Makhoul A., "Performance study of multiple cover-set strategies for mission-critical video surveillance with wireless video sensors," IEEE 6th International Conference on Wireless and Mobile Computing, Networking and Communications (WiMob 2010), 11-13 Oct. 2010, Niagara Falls, Canada. http://dx.doi.org/10.1109/WIMOB.2010.5644991

[17] Evalvid, "Evalvid - a video quality evaluation tool-set," available at: http://www2.tkn.tuberlin.de/research/evalvid/fw.html (last accessed June 30, 2012)

[18]A. Boulis, "Castalia - A simulator for Wireless Sensor Networks and Body Area Networks," version 2.2, User's. Manual, October 2009. At http://castalia.npc.nicta.com.au/pdfs/Castalia\%20-\%20User\%20Manual.pdf (last accessed June 30, 2012)

[19] Video Trace Library, "Yuv video sequences," available at: http://trace.eas.asu.edu/yuv/ (last accessed June 30, 2012)

[20] Alemdar H., Ersoy C., "Wireless sensor networks for healthcare: A survey," Computer Networks, vol. 54, no. 15, pp. 2010. http://dx.doi.org/10.1016/j.comnet.2010.05.003

[21] Aguiar E., Riker A., Abelém, A. J., MU, M. and Cerqueira E. "Video quality estimator for wireless mesh networks," in 20th International Workshop on Quality of Service (IEEE/ACM IWQoS 2012), Coimbra, 2012. http://dx.doi.org/10.1109/IWQoS.2012.6245980

\section{Copyright Disclaimer}

Copyright reserved by the author(s).

This article is an open-access article distributed under the terms and conditions of the Creative Commons Attribution license (http://creativecommons.org/licenses/by/3.0/). 